International Journal of Pure and Applied Mathematics

Volume 105 No. 4 2015, 715-721

ISSN: 1311-8080 (printed version); ISSN: 1314-3395 (on-line version)

url: http://www.ijpam.eu

doi: http://dx.doi.org/10.12732/ijpam.v105i4.11

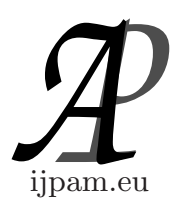

\title{
ON A SEQUENCE OF TRIDIAGONAL MATRICES, WHOSE PERMANENTS ARE RELATED TO FIBONACCI AND LUCAS NUMBERS
}

\author{
Ivana Matoušova ${ }^{1}$, Pavel Trojovský $^{2} \S$ \\ ${ }^{1,2}$ Department of Mathematics \\ Faculty of Science \\ University of Hradec Králové \\ Rokitanského 62 \\ 50003 Hradec Králové, CZECH REPUBLIC
}

\begin{abstract}
In this paper, we generalize result on connection permanents of special tridiagonal matrices with Fibonacci numbers, as we show that more general sequences of tridiagonal matrices is related to the sequence of Fibonacci numbers.
\end{abstract}

AMS Subject Classification: 11A51, 11A07, 11Y11

Key Words: recurrence, Fibonacci number, Lucas number, tridiagonal matrix, permanent

\section{Introduction}

The Fibonacci sequence (or the sequence of Fibonacci numbers) $\left(F_{n}\right)_{n>0}$ is the sequence of positive integers satisfying the recurrence $F_{n+2}=F_{n+1}+F_{n}$ with the initial conditions $F_{0}=0$ and $F_{1}=1$. Similarily the Lucas numbers are the sequence of integers $\left(L_{n}\right)_{n \geq 0}$ defined by the recurrence relation $L_{n+2}=$ $L_{n+1}+L_{n}$, with $L_{0}=2$ and $L_{1}=1$. The Fibonacci and Lucas numbers are well-known for possessing many amazing properties (see e. g. [6], [11] or [12]). For example the following identities hold between the Fibonacci and Lucas

Received: September 4, 2015

(C) 2015 Academic Publications, Ltd.

$\S$ Correspondence author url: www.acadpubl.eu 
numbers

$$
\begin{aligned}
F_{n+2} & =2 F_{n}+F_{n-1}, \\
L_{n+1} & =3 F_{n}+F_{n-1}, \\
L_{n} & =F_{n}+2 F_{n-1} .
\end{aligned}
$$

Square matrix $\mathbb{A}=\left(a_{j k}\right)$ of the order $n$, where $a_{j k}=0$ for $|k-j|>1$ and $1 \leq j, k \leq n$, is called tridiagonal matrix. Let $\mathbb{B}=\left(b_{j k}\right)$ be any square matrix of the order $n$. The permanent of matrix $\mathbb{B}$ is defined by the following way

$$
\operatorname{per} \mathbb{B}=\sum_{\sigma \in S_{\mathrm{n}}} \prod_{j=1}^{n} b_{j \sigma(j)},
$$

where the summation extends over all permutations $\sigma$ of the symmetric group $S_{n}$. The permanent of a matrix is analogous to the determinant, where all of the signs used in the Laplace expansion of minors are positive (see [8]).

Arguably, the first result on the relation between the permanent of tridiagonal matrix and the Fibonacci numbers can be extracted from a more general case, which is due to Minc [8], but this result was exactly given by King and Parker [5]. They derived that for permanent of the tridiagonal matrix

$$
\mathbb{C}(n)=\left(\begin{array}{ccccc}
1 & 1 & 0 & \cdots & 0 \\
1 & 1 & 1 & \ddots & \vdots \\
0 & 1 & 1 & \ddots & 0 \\
\vdots & \ddots & \ddots & \ddots & 1 \\
0 & \cdots & 0 & 1 & 1
\end{array}\right),
$$

where $n$ is order of this matrix, the following holds

$$
\operatorname{per} \mathbb{C}(n)=F_{n+1} .
$$

Kiliç and Taşci [3] found, using contraction method of a square matrix (Brualdi and Gibson [1] introduced this method), that for permanent of the matrix

$$
\mathbb{D}(n)=\left(\begin{array}{ccccc}
1 & -1 & 0 & \cdots & 0 \\
-1 & 1 & -1 & \ddots & \vdots \\
0 & -1 & 1 & \ddots & 0 \\
\vdots & \ddots & \ddots & \ddots & -1 \\
0 & \cdots & 0 & -1 & 1
\end{array}\right)
$$


the following holds

$$
\operatorname{perD}(n)=F_{n+1} \text {. }
$$

Jína and Trojovský [2] generalized results (4) and (5), as they showed that for permanent of the matrix

$$
\mathbb{E}(n)=\left(\begin{array}{ccccc}
1 & \frac{1}{x} & 0 & \cdots & 0 \\
x & 1 & \ddots & \ddots & \vdots \\
0 & x & \ddots & \frac{1}{x} & 0 \\
\vdots & \ddots & x & 1 & \frac{1}{x} \\
0 & \cdots & 0 & x & 1
\end{array}\right)
$$

where $x \neq 0$, the following holds

$$
\operatorname{per} \mathbb{E}(n)=F_{n+1}
$$

for $n \geq 1$.

Another recent interesting application of the tridiagonal matrices can be found for example in $[9,10]$. In this paper we turn our attention to the relation of permanents of special tridiagonal matrices with Fibonacci numbers. We show that matrix (6) can be generalized by a matrix, whose permanent is related to Fibonacci numbers too.

\section{Preliminary Results}

We will use the following lemma from [3], which can be easily proved by Laplace expansion for permanents.

Lemma 1. (Lemma 3 of $[3])$ Let $\{\mathbb{F}(n), n=1,2, \ldots\}$ be the sequence of matrices of type $n \times n$ in the following form

$$
\mathbb{F}(n)=\left(\begin{array}{ccccc}
f_{1,1} & f_{1,2} & 0 & \cdots & 0 \\
f_{2,1} & f_{2,2} & f_{2,3} & \ddots & \vdots \\
0 & f_{3,2} & f_{3,3} & \ddots & 0 \\
\vdots & \ddots & \ddots & \ddots & f_{n-1, n} \\
0 & \cdots & 0 & f_{n, n-1} & f_{n, n}
\end{array}\right)
$$

Then the successive permanents of sequence $\mathbb{F}(n)$ are given by recursive formula 


$$
\begin{aligned}
\operatorname{per} \mathbb{F}(1) & =f_{1,1} \\
\operatorname{per} \mathbb{F}(2) & =f_{1,1} f_{2,2}+f_{1,2} f_{2,1} \\
\operatorname{per} \mathbb{F}(n) & =f_{n, n} \operatorname{per} \mathbb{F}(n-1)+f_{n-1, n} f_{n, n-1} \operatorname{per} \mathbb{F}(n-2) .
\end{aligned}
$$

\section{Main Results}

Theorem 2. Let $x$ be any positive integer. Let $\left(\varepsilon_{n}\right)_{n \geq 0},\left(\delta_{n}\right)_{n \geq 0}$ be any sequences of complex numbers, with property $\varepsilon_{k} \delta_{k}=1$ for any $k, 1 \leq k \leq n$. Let $\left\{\mathbb{G}_{n}^{\alpha}(x), n=1,2,3, \cdots \wedge \alpha \in\{0,1\}\right\}$ be a sequence of tridiagonal matrices in the form

$$
g_{j k}^{\alpha}(x)=\left\{\begin{array}{cc}
x^{\alpha}, & j=k=1 ; \\
x^{1-\alpha}, & j=k=2 ; \\
1, & j=k \wedge j \geq 3 ; \\
\varepsilon_{j}, & k=j+1 ; \\
\delta_{k}, & k=j-1 ; \\
0, & \text { otherwise }
\end{array}\right.
$$

i. e.

$$
\mathbb{G}_{n}^{\alpha}(x)=\left(\begin{array}{cccccc}
x^{\alpha} & \varepsilon_{1} & 0 & \cdots & \cdots & 0 \\
\delta_{1} & x^{1-\alpha} & \varepsilon_{2} & \ddots & \ddots & \vdots \\
0 & \delta_{2} & 1 & \ddots & \ddots & \vdots \\
\vdots & \ddots & \ddots & \ddots & \varepsilon_{n-2} & 0 \\
\vdots & \ddots & \ddots & \delta_{n-2} & 1 & \varepsilon_{n-1} \\
0 & \cdots & \cdots & 0 & \delta_{n-1} & 1
\end{array}\right) .
$$

Then

$$
\operatorname{per} \mathbb{G}_{n}^{\alpha}(x)=F_{n} x^{\alpha}+F_{n-1} x^{1-\alpha}= \begin{cases}F_{n}+F_{n-1} x, & \alpha=0 \\ F_{n-1}+F_{n} x, & \alpha=1 .\end{cases}
$$

Proof. We use mathematical induction with respect to $n$. For $n=1$ and $n=2$ we have

$$
\begin{aligned}
\operatorname{per} \mathbb{G}_{1}^{\alpha}(x) & =g_{1,1}(x)=x^{\alpha}=F_{1} x^{\alpha}+F_{0} x^{1-\alpha} \\
\operatorname{per} \mathbb{G}_{2}^{\alpha}(x) & =g_{1,1}(x) g_{2,2}(x)+g_{1,2}(x) g_{2,1}(x) \\
& =x+\varepsilon_{1} \delta_{1}=x+1=x^{\alpha}+x^{1-\alpha} \\
& =F_{2} x^{\alpha}+F_{1} x^{1-\alpha}
\end{aligned}
$$


respectively, hence relation (8) holds. Suppose that the assertion holds for every $k, 3 \leq k<n$ Then we have to show that the assertion is true for $n$ too. We obtain by identity $(7)$

$$
\begin{aligned}
\operatorname{per} \mathbb{G}_{n}^{\alpha}(x) & =g_{n, n}(x) \operatorname{per} \mathbb{G}_{n-1}^{\alpha}(x)+g_{n-1, n}(x) g_{n, n-1}(x) \operatorname{per} \mathbb{G}_{n-2}^{\alpha}(x) \\
& =1 \cdot \operatorname{per} \mathbb{G}_{n-1}^{\alpha}(x)+\varepsilon_{n-1} \delta_{n-1} \operatorname{per} \mathbb{G}_{n-2}^{\alpha}(x) \\
& =\operatorname{per} \mathbb{G}_{n-1}^{\alpha}(x)+\operatorname{per} \mathbb{G}_{n-2}^{\alpha}(x)
\end{aligned}
$$

for $n \geq 3$. The induction follows for $n \geq 3$ if we assume that (8) holds when $n$ is replaced by $n-2$ or $n-1$

$$
\begin{aligned}
\operatorname{per} \mathbb{G}_{n}^{\alpha}(x) & =\left(F_{n-1} x^{\alpha}+F_{n-2} x^{1-\alpha}\right)+\left(F_{n-2} x^{\alpha}+F_{n-3} x^{1-\alpha}\right) \\
& =F_{n} x^{\alpha}+F_{n-1} x^{1-\alpha} .
\end{aligned}
$$

Hence (8) holds for $n$ as well and the assertion is proved.

Corollary 3. Setting $x=1$ and $\varepsilon_{k}=\delta_{k}=1$ or $x=1$ and $\varepsilon_{k}=\delta_{k}=-1$ in Theorem 2, for $1 \leq k \leq n$, we directly obtain (4) or (5) respectively.

Similarly we can obtain infinitely many interesting $n$-square matrices, whose permanents are equal to the Fibonacci or Lucac numbers, using Theorem 2, but there are integer matrices of this type only for entries $\varepsilon_{k}= \pm 1$, $\delta_{k}=\varepsilon_{k}$, where $1 \leq k \leq n$. For example, we obtain the following sequences of integer matrices (the assertions follow from identities (1), (2) and (3))

$$
\operatorname{per} \mathbb{G}_{n}^{1}(2)=\left(\begin{array}{cccccc}
2 & 1 & 0 & \cdots & \cdots & 0 \\
1 & 1 & 1 & 0 & \ddots & \vdots \\
0 & 1 & 1 & \ddots & \ddots & \vdots \\
\vdots & \ddots & \ddots & \ddots & \ddots & 0 \\
\vdots & \ddots & \ddots & \ddots & \ddots & 1 \\
0 & \cdots & \cdots & 0 & 1 & 1
\end{array}\right)=F_{n+2}
$$




$$
\begin{aligned}
\operatorname{per} \mathbb{G}_{n}^{1}(3)= & \left(\begin{array}{cccccc}
3 & 1 & 0 & \cdots & \cdots & 0 \\
1 & 1 & 1 & 0 & \ddots & \vdots \\
0 & 1 & 1 & \ddots & \ddots & \vdots \\
\vdots & \ddots & \ddots & \ddots & \ddots & 0 \\
\vdots & \ddots & \ddots & \ddots & \ddots & 1 \\
0 & \cdots & \cdots & 0 & 1 & 1
\end{array}\right)=L_{n+1}, \\
\operatorname{per} \mathbb{G}_{n}^{0}(2)= & \left(\begin{array}{cccccc}
1 & 1 & 0 & \cdots & \cdots & 0 \\
1 & 2 & 1 & 0 & \ddots & \vdots \\
0 & 1 & 1 & \ddots & \ddots & \vdots \\
\vdots & \ddots & \ddots & \ddots & \ddots & 0 \\
\vdots & \ddots & \ddots & \ddots & \ddots & 1 \\
0 & \cdots & \cdots & 0 & 1 & 1
\end{array}\right)=L_{n} .
\end{aligned}
$$

\section{Acknowledgements}

The author thanks Specific research PřFUHK2015 for financial support.

\section{References}

[1] R. A. Brualdi, P. M. Gibson, Convex polyhedra of doubly stochastic matrices. I. Applications of permanent function, J. Combin. Theory (A) 22, (1977), 194-230.

[2] J. Jína, P. Trojovský, On permanents of some tridiagonal matrices connected with Fibonacci numbers. Int. J. Pure and Appl. Math. 97 (1), (2014), 79-87.

[3] E. Kiliç, D. Taşci, On the permanents of some tridiagonal matrices with applications to the Fibonacci and Lucas numbers, Rocky Mountain J. Math. 37, No. 6 (2007), 1953-1969.

[4] K. Kaygisiz, A. Şahin, Determinant and Permanent of Hessenberg Matrix and Fibonacci Type Numbers, Gen. Math. Notes, 9, No. 2 (2012), 32-41

[5] B. W. King, F. D. Parker, Fibonacci matrix and the permanent function, Fib. Quart., 7, No. 5 (1969), 539-544. 
[6] T. Koshy, Fibonacci and Lucas Numbers with Applications, Wiley, New York, 2001.

[7] H. Minc, Permanents of (0,1)-circulants, Canad. Math. Bul. 7, (1964), 253-263.

[8] H. Minc, Encyclopedia of Mathematics and Its Applications, Permanents, Vol. 6, Addison-Wesley Publishing Company, London, 1978.

[9] A. Ohashi, T. S. Usuda, T. Sogabe, F. Ylmaz, On tensor product decomposition of $k$-tridiagonal Toeplitz matrices Int. J. Pure and Appl. Math. 103 (3), (2015), 537-545 .

[10] P. Trojovský, On a sequence of tridiagonal matrices whose determinants are Fibonacci numbers $F_{n+1}$. Int. J. Pure and Appl. Math. 102 (3), (2015), $527-532$.

[11] S. Vajda, Fibonacci \& Lucas Numbers, and the Golden Section: Theory and Applications, Ellis Horwood, Chichester, 1989.

[12] N. N. Vorobiev, Fibonacci Numbers, Birkhäuser, Basel, 2003.

[13] F. Yllmaz, T. Sogabe, A note on symetric k-tridiagonal matrix family and the Fibonacci numbers, Int. J. Pure and Appl. Math., 96, No. 2 (2014), 289-298.

[14] F. Yilmaz, D. Bozkurt, On the Fibonacci and Lucas numbers, their sums and permanents of one type of Hessenberg Matrices, arXiv:1302.0668 [math.NT] (2013). 
